\title{
Informational Model of Consciousness: From Philosophic Concepts to an Information Science of Consciousness
}

\author{
Florin Gaiseanu \\ Science and Technology of Information Bucharest (Romania) and Barcelona (Spain)
}

\begin{abstract}
On the long and well-worn road of many, but justifiable attempts of human to discover his origin, his trajectory as a species, and a suitable understanding consciousness, his system allowing the connection to the environment and to his own organism, the concepts and models of philosophy enunciated or experienced by millennia, meet today with modern science concepts of physics and of science of information. Based on recent discoveries of quantum physics and astrophysics, revealing a new understanding of our environment and starting from some philosophical concepts on information of matter and of living structures, this work discusses the dynamics of information within the frame of the Informational Model of Consciousness as an informational system of the human body, connected both to the environment and to the body itself, to control the adaptation for survival. It is shown that consciousness is actually an informational projection in the mind of seven informational subsystems, three of which forming the operative system of consciousness for the short-term adaptation, and other three forming the programmed operating system, dedicated to the maintenance of body and to the long-term survival of species, showing various inputs and outputs of information. The seventh subsystem is the information pole, connecting the organism with the external information, especially related to the extra-sensorial properties of the mind, the human body appearing as a bipolar info-matter structure, managed by the brain. The received information is progressively integrated into the informational system of the organism, which absorbs and emanates information as a reactive system for adaptation, able to operate both with matter-related (codified) and non-matter related (virtual) information. As both connections with external and internal environment (body itself) can be described in terms of information, this model opens the gate to investigate consciousness by means of the tools of the information science, offering also answers to the philosophic "mind-body" problem and to the "hard" problem and showing correspondences with some ancient philosophies.
\end{abstract}

Keywords: consciousness, informational model of consciousness, dynamics of information, matter integration of information, information science, philosophical concepts, matter-related information, information-assisted living

\section{Introduction}

Since ancient times, the humanity has contemplated his relationship with the universe and with itself existence, wondering "who we are", "how our body/mind works", and "where we are going to". Understanding consciousness has been and still remains an issue of debate in this broad context (Gaiseanu, 2018b; 2018d; 2019b). From the perspective of the society which we live in today, flooded by the high technology products of micro-electronics and micro-systems, making our lives easier and substituting many of our hard activities, but

Florin Gaiseanu, Ph.D., professor, Science and Technology of Information Bucharest (Romania) and Barcelona (Spain). 
mostly enabling the deep investigation of matter and of our universe, seems to be incredible the intuition of our ancestors, who have been able to decipher some of the mysteries of the immense unknown. If we only look at the great advance that the Greek philosophers have recorded on the conception about consciousness, referring for instance only to Aristotle and Plato, it seems amazing how the human mind itself has succeeded to penetrate the unknown and scrutinize the horizons of knowledge (Gaiseanu, 2019b). Mihai Draganescu, a prominent scientist and philosopher, dedicated to the development of science and technology of information, but also contributing with valuable concepts in the field of philosophy of matter and information, often referred in his works to Greek philosophy, particularly to Aristotle's conception on how the environment is reflected in consciousness, which is still a valid source of knowledge and inspiration (Draganescu, 1979; 1990). For instance, Aristotle believed that the reception of the surrounding reality in mind is done by association with something a priori known, which was the basis of the development of applications not only in neural network systems, but also in understanding of such specific mechanisms of consciousness (Perlovski, 2001). Philosophy has therefore been and still remains a useful tool of exploration and knowledge, contributing to the design of some directions for the scientific investigations.

However, while philosophy is dedicated to discuss and analyze consciousness especially from a phenomenological point of view, the "hard" sciences have the role to discover the intimate operating mechanisms. Therefore, as much as precious could be this contribution, as much it is necessary the study of matter that surrounds us, in its diversity of forms and laws and of the universe where we live in, by means of some other disciplines, especially by physics, and more recently by information science (Gaiseanu, 2018d). The symbiosis between philosophy and the "hard" sciences is therefore irreplaceable. That is why the analysis of consciousness remained not only the attribute of philosophy, but also was converted into the investigation field of many other scientific branches, including, but not restricting to neurology, psychiatry, psychology, sociology, pharmacology, anesthesiology, more recently to information science (Gaiseanu, 2018d) and to physics, which had and has a determining role in discovering the mechanisms and laws of nature, and which is still appealed to bring more contributions to understanding the phenomena associated with consciousness and to provide the necessary experimental evidence.

The development of the investigation systems of the micro- and macro- scale world allowed remarkable discoveries, concerning the behavior both of quantum particles and of the structure and composition of the universe. These results can directly reinforce the investigations on consciousness, from the perspective both of microscopic and macroscopic/cosmic phenomena. That is because we cannot separate the human from the environment in which he lives, this being an inseparable part of it (Gaiseanu, 2019d). On this basis, the more and more increasing of the accumulating data and with participation of the informational concepts, the information science and technology increases its intervention also in this field, starting from the basic concepts on matter-information and information-based living structures (Draganescu, 1990; Gaiseanu, 2016a), questions raised from the philosophic approach of consciousness and its basic support. The "mind-body problem" (Haward, 2016) and its derivatives, like the so called "hard problem” (Chalmers, 1995), could find answers on the base of informational approach of consciousness, as it will be discussed in this paper.

Over time, various models of consciousness have been elaborated and developed, each of them based only on one or more particular aspects of consciousness. Reviewing all of these models is a difficult task, and it is not the goal of this article. However, taking into account the main results reported to date, it is useful to emphasize the following conclusions: (i) consciousness cannot be described taking into account only the 
electrical activity of the brain, and which allowed the correlation between low frequency currents through brain nerve cells and thoughts, without to include the quantum physics phenomena (Humeroff, 1998; Hameroff \& Penrose, 1996, 2014; Radin, 2006); (ii) the phenomena associated with near-death experiences (NDEs), which have become the basis of a rigorous scientific investigation of the consciousness "extra"-properties, overpass the current scientific understanding (Fracasso \& Friedman, 2011), and quantum mechanisms should be involved to properly approach them (Gaiseanu, 2017b; 2017c); (iii) the extra-properties phenomena, such as remote communication by mind (Radin, 2006), premonition and psychokinesis (Ignatenko, 2006), to name only the most widely known, cannot be explained by the current models, without resorting to the latest scientific conquests on the intimate structure of matter and to that of the universe which we live in (Gaiseanu, 2016a). So, although various models of consciousness have been proposed, some of them suggesting that an informational modeling could consistently explain the properties of mind (Chalmers, 1995; Van Lammel, 2006), only recently a coherent modeling began to be concretized (Tononi, 2004; Gaiseanu, 2017c, 2018d, 2019c, 2019d).

The aim of this work is to show that the Informational Model of Consciousness (IMC), based on recent investigation results referring to the nature of the universe and particle behavior in quantum physics (Gaiseanu, 2016a; 2016b; 2017b; 2017c; 2018d), is able to fully describe consciousness and its properties in terms of information and its dynamics within the informational system of the organism, offering response to philosophic problems on consciousness and pointing out in the same time the contribution of philosophic concepts on information earlier reported, as a basic statement for the development today of an informational science of consciousness.

\section{Informational Model of Consciousness and Dynamics of Information}

\section{The Informational Structure of Consciousness}

The latest discoveries on physics of particles (Aharonov, Popescu, Rohrlich, \& Skrzypczyk, 2013; Denkmayr et al., 2013) and astrophysics (Hajdukovic, 2010; 2011; 2012), allowed a remarkable advance in the understanding of consciousness (Gaiseanu, 2016a; 2016b; 2018a; 2018b; 2018c; 2018d). The introduction and substantiation of the concept of information as a component of matter, which can be revealed separately of it, as demonstrated during some recent experiments in quantum physics (Denkmayr et al., 2013; Aharonov et al., 2013) and as predicted by the philosopher and scientist Draganescu (1979; 1990; 2004), allowed to elaborate a new and original consciousness information model (Gaiseanu, 2017b; 2017c; 2018b; 2019c). The analysis and identification of the information subsystems of consciousness, defined by their distinct but integrated functions, informationally sustained, revealed the existence of seven informational cognitive centers, as shown in Figure 1. These centers represent the projection in consciousness of the specific components of the informational system of the human body, which can be presented as follows. 


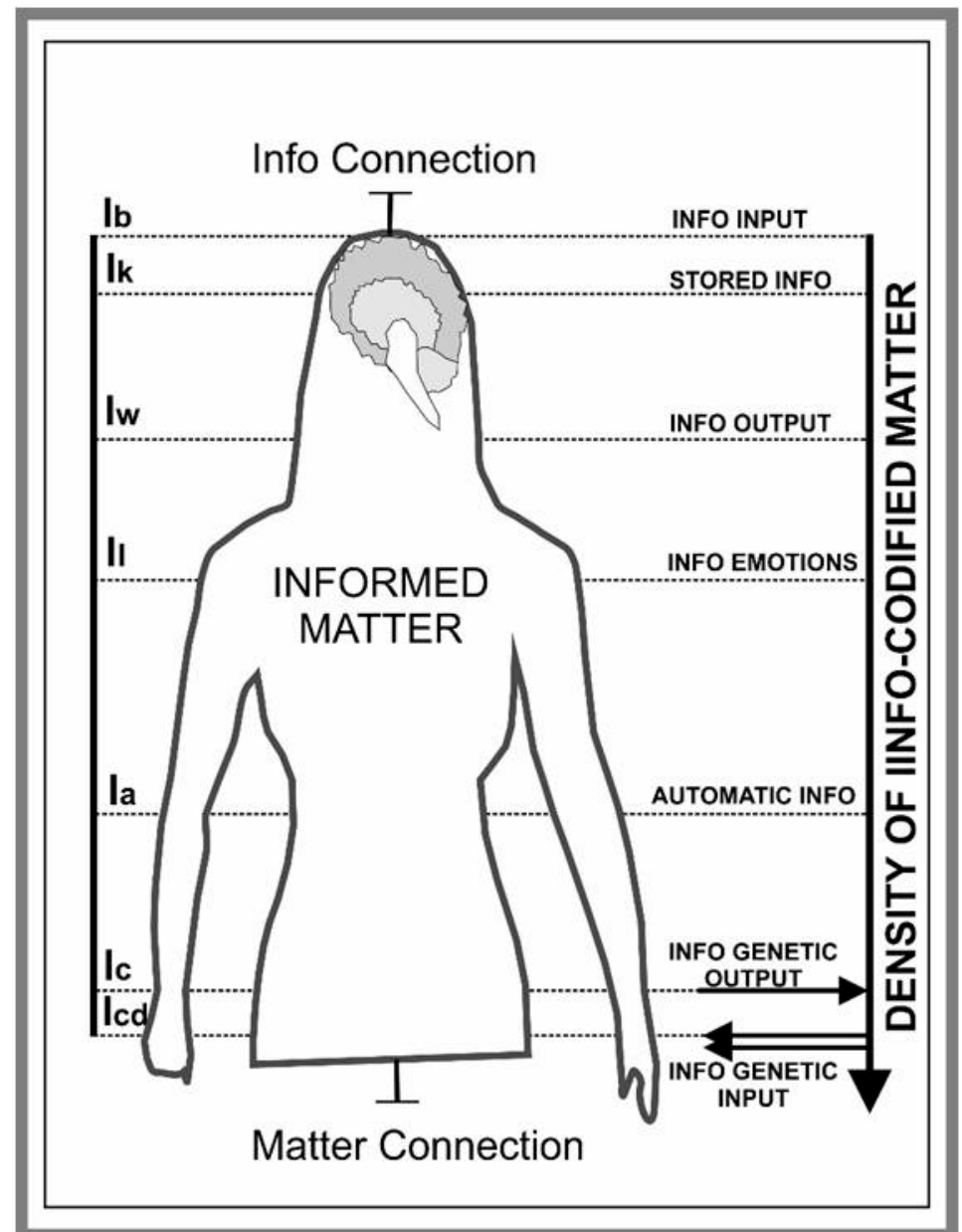

Figure 1. Consciousness as an integrated informational system, represented by means of informational components, reflected at the conscious level by the corresponding cognition centers, suggestively defined by: IC => Ibelieve (= Ib), CASI $=>$ Iknow (= Ik), CDC => Iwant (= Iw), IES => Ilove (= Il), MIS => Iam (= Ia), GTS => Icreate (= Ic), IGG => Icreated $(=\mathrm{Icd})$. The arrow indicates the increase of the integration degree of information from input "free"-non-matter-related (Ib, Ik), to matter-related information, with a maximum as genetic codified information (Ic, Icd).

(1) Info-Connection (IC), which provides the access to the informational field of the universe, explaining the extra-sensorial phenomena (ESP) experimentally observed (Meijer, 2013b) and recently discussed (Gaiseanu, 2016a; 2018d). The projection of this connection in consciousness is represented by the cognitive center suggestively called Ibelieve (Ib).

(2) Center of Acquisition and Storage of Information (CASI), defined as the sum of all brain areas and adjacent circuits connected to information sensors (Gaiseanu, 2019c), dedicated to the acquisition and storage of information. The projection in consciousness is defined as a cognitive center Iknow (Ik), consisting in memory.

(3) Center of Decision and Command (CDC), composed by summing all brain areas responsible for the processing of information and the elaboration of the decision (Gaiseanu, 2019c). The corresponding cognitive center is suggestively called Iwant (Iw), reflecting the function of decision and command.

(4) Info-Emotional System (IES), responsible for the info-emotional activity, as a reaction of the organism (defined as informed matter [IM]), to the detected information. The corresponding cognitive center is 
suggestively called Ilove (Il), reflecting the reaction with respect to the informational input (acceptance/rejection), as a resonance oscillator.

(5) Maintenance Information System (MIS), assuring the individual body survival by connection with matter (food, water, and air). The corresponding cognitive center in consciousness is suggestively called Iam (Ia), reflecting the self-status, health, and actually the power (energy) of the organism.

(6) Genetic Transmission System (GTS), which ensures the transmission of genetic (matter-codified) information to the subsequent generations, so the survival of species in the future. The associated cognitive center is suggestively called Icreate (Ic), responsible for the creation/formation of the new generation (Gaiseanu, 2018c).

(7) Info-Genetic Generator (IGG), which assures the development of the body according to the specific age, from birth to extinction. The associate cognitive center Icreated (Icd) is reflected in consciousness by the inherited predispositions, mentality, talents, and abilities.

\section{External and Internal Info-Connectivity and Informational Dynamics}

All these systems defined above are managed by the brain. The external information, which is detected by our senses, i.e., seeing, hearing, smell, taste, and touch, is captured by the external sensors, while the internal information, such as hunger, thirst, pain, and emotion is captured by the internal sensors and transmitted to the brain through specific, associated informational circuits. The received information from sensors is the information input in the informational system, as it is marked in Figure 1 as a top line. The storage of this information is done in the Center of Acquisition and Storage of Information (CASI), which is just the memory. CASI memorizes the entire life experience of the individual, which is ordered in such way to become a reference for the new experiences. Indeed, once the "good" and the "bad" have been tested, the two bipolar alternatives will be used as decision criteria for the future (Yes/No) decisional selections (Gaiseanu, 2018c; 2019a). The attitude, operated by CDC, is actually the information output of our operative (conscious) informational system, used for the adaptation to the external challenges and internal needs, assuring in this way the survival requirements.

The decision is defined as a result of the activity of the Center of Decision and Command (CDC), which processes the received information, elaborates the decision and transmits it to the execution systems, represented in particular by the muscles and also by other specific execution components. It is perceived in consciousness by Iw as the attitude and is expressed especially through the speaking system. The attitude is actually the output information for adaptation to the environment and personal needs, and it is a function not only of the information received from sensors and stored in CASI, but also of the decision criteria, which are acquired essentially from the "Yes/No" type experience gained during the life (Gaiseanu, 2018c), and from the genetic informational input transmitted by the parents. CASI is reflected in consciousness by Ik, while IGG through Icd and manifested by skills, predispositions, and talents, and also by the acquired experience as education during the first years of life. The experience within the family consisting in cultural, religious, and social heritage is fundamental to the formation of the future adult, the concepts, and habits acquired during this period becoming evaluation and decision criteria throughout the entire life.

An important component in the elaboration of the decision is emotion, operated by Il and attributed to the Info-Emotional System (IES). Indeed, emotion is a specific intrinsic state as a reaction of the organism (informed matter [IM]) with respect to the received information processed by the Center of Decision and 
Command (CDC). Therefore, a final decision expressed by the attitude is most often elaborated as a function of this state and not only as a result of the logical processing in CDC (Gaiseanu \& Graur, 2018). The actual health status of the body, monitored by the Maintenance Informational System (MIS) and reflected in the conscious mind through Ia, may also intervene in the decision process, because this determines actually the power of execution. The Genetic Transmission System (GTS) and reflected in the conscious mind through Ic, expressing the level and the quality of the external relationships, is also an important factor in the decisional process, because it is actually a direct indicator of the socialization-related status.

The Info-Connection (IC) ensures the stability, the equilibrium of the body and mind and is implied in the Extra-Sensorial Phenomena (ESP), in Near-Death Experiences (NDEs) (Gaiseanu, 2017a), Religious and Mystical Experiences (RMEs), and in the so called para-Psychological (PSY) phenomena (Gaiseanu, 2019d). The connection can be felt in consciousness by Ib, as an extra-power, to be used especially during the difficulty moments as a health insurance, inspiring to body (informed matter) trust in the forces of life. That is why Ib also contributes to the decision process, the attitude being ultimately the result of the decision by the co-participation of all information centers (Gaiseanuand \& Graur, 2018; Gaiseanu, 2019a).

We called Ilove (Il) the reflection of Info-Emotional System in the conscious mind, because the human being is naturally willing to relate himself to the environment in which he lives under harmonious conditions, with joy of living. Love is actually a form of association with the object/subject of desire, and the natural desire of man is to associate himself with the environment to which he belongs, in order to know it and to adapt to it. Love is also an extremely powerful factor of associating information with our body, even though we are not always aware of this. Love is from this point of view a fundamental binding force between information and matter. If CASI and CDC are systems mainly related to virtual information, love is primarily a force that tends to unite matter with information. Actually, the association is a fundamental property driving the information-assisted structuring. This property is also reflected in the perception and memorization, the new information being added to those similar, already consolidated in memory. That is probably why the great thinkers and philosophers of life who have defined and preached fundamental philosophical concepts became religions, have pointed out and emphasized the role of love as a binder and helper of life, against confrontation, which is a force of dissociation and destruction. Explaining now this in terms of modern physics, we can say that love is an force which unifies information with matter and it is opposed to disorder and destruction dictated by the entropic forces of odium and vengeance.

Under the light of the above comments, we can refer to the correlated activity expressed by the sum (CASI + CDC + IES) as to an Operational Information System (OIS), which ensures the adaptation to the environmental dynamics on short and medium term. This system is reactive and anti-entropic, because it is a response of the living structure to the environmental challenges, aimed to ensure the continuity of life, and thus the survival of the body. The decision is a result of a continuous "Yes/No" activity reflected by Iw, even though these elemental decision steps may be as small as possible. A "Yes" step can be followed by a "Not" step and the effective advance may be zero, actually ensuring a neutrality or equilibrium state. The dual character of the universe, represented by bipolar binary forces like matter/antimatter, gravity (entropy)/anti-gravity (anti-entropy), action/reaction, and attraction/rejection, can be recognized also by "Yes/No" unit in the operating mode of the human decisional system.

Unlike OIS, the Programmed Information System (PIS) defined as (MIS + GTS + IGG) operates automatically, without the intervention of the conscious mind. MIS mainly ensures both the transformation of 
nourishment (food, water, and air) into the material components necessary for life, and the distribution of nutriments to the cells. So, this system represents practically the connection with the non-living external matter, integrating it under suitable conditions into Informed Matter, the body. Driven by the brain, this activity therefore supports the "hard" part of the informational system and is especially associated with the digestive and cardiovascular operational structures. The internal sensors transmit to the brain the corresponding sensations, like hunger, thirst, and the need of oxygen, reflected in the conscious mind by the center Ia. This center therefore is correlated with the perception and evaluations of the personal needs and status, referring especially to the state of health and general power of the organism, because MIT is actually its primary energy source. From this point of view, we can consider MIT as a system directly connected to non-living matter, as IC is the information pole of the organism. Therefore, the human being appears to be a bipolar info-matter system.

The Genetic Transmission System (GTS) ensures the functioning of the components dedicated to the preparation and transmission of the genetic codified (matter-related) information, and is projected in consciousness at the conscious level through Ic, especially by procreation impulses. In turn, IGG, reflected in consciousness through Icd, is expressed by predispositions and mentality, as genetic informational dowry inherited from parents. This system also drives the development and involution of body according to the age. IGG represents actually the genetic information input of the organism, and GTS is the info-genetic output, ensuring the perpetuation of the species, as a form of long-term survival. PIS is assimilable with the subconscious mind, while OIS with the conscious level of consciousness.

From the previous description, we can see that these systems refer, on the one hand, to the transformation of ordinary input matter into sub-components of matter, energy, and information, in order to support and recompose the Informed (living) Matter (by MIS), and, on the other hand, to the encoding (by GTS)/decoding (by IGG) of information in and from Informed Matter, assuring the continuity of life within two consecutive generations. These systems, a heritage of species evolution, fully (so completely) cover the entire time horizon of life, namely the past (by IGG), the present (through MIS), and the future (through GTS). PIS operates with matter by means of all its main components (energy, information, and matter itself), information being integrated into matter by a codification process. The codified information in PIS can be therefore referred as ta a matter-related information.

The connectivity with the external sources of information is assured basically by the Info-Connection system IC: when the external sensors do not work, disconnecting the organism from the reality, the extra-sensorial signals could be easier detected. In a larger, more general meaning, IC includes therefore all categories of signals. The info-connectivity with the body itself is related by the activity of the internal sensors (Gaiseanu, 2019c). Analyzing the integration degree of information and relating it with the informational subsystems, we have to observe therefore that starting from IC, where the "free" (non-matter-related) information is received, and going down (see Figure 1) to the next subsystems, the integration degree of information is increased to a maximum represented by the genetic information. Indeed, the acquired information is more and better integrated by repetition, one converting it into automatic, prototype chains by means of epigenetic processes, becoming finally and definitely genetic information, which allows the acquired traits transmission to the next generation (Gaiseanu, 2019c). The internal transmission of information is supported by both electrical (through nervous cells) and non-electrical (embodiment/disembodiment) mechanisms of information, as it was recently shown (Gaiseanu, 2019c). These processes demonstrate the info-nature of the organism, acting as an informational processor, and show the intimate relation between mind 
and body, offering answers to the philosophic "mind-body" problem and to the derivative "hard" problem, referring to the correlation between the physical nature and sensations (Chalmers, 1995). While the input genetic information allows the development of the new organism by cellular division and differentiation, the acquired information can be transmitted as an info-genetic output. On the other hand, the info-life experience tends to separate during NDEs from the body, as a final disembodiment process (Gaiseanu, 2017b).

\section{From Philosophy to an Information Science of Consciousness}

The philosophical hypotheses of Mihai Draganescu (1990) proved to be correct, validated by science. According to Draganescu's philosophical vision, expressed especially in his book Information of Matter, the material structures cannot be formed without the contribution of an essential component of the universe, which is information. To refer to these concepts, let us first of all clarify what information does means.

In the info-communication systems based by electronics and microelectronics, information is defined in Shannon's (1948) terms, as a measure of the certainty of choosing between a finite number of alternatives. Extracting information from a system means the reduction of its entropy, making the difference between the uncertainty (entropy) of the system when its state is unknown, and that of the system, when its state is known. This method has been extensively used to illustrate how the brain could actually operate, changing information between its various inferior and superior levels (Tononi, 2004). However, as it was pointed out above, the theory of information can be also applied to the transmission/reception of information in the informational system of the organism (Gaiseanu, 2019c). Information is not only a philosophic concept, it is a physical parameter of matter, both of non-living and living structures, as it is presented in more details below.

\section{Information Assisting the Matter Structuration}

Within the philosophical model of matter, according to Draganescu's (1990) conception, a basic, ultimate species of matter that he called "deep" matter is incapable to structure itself. The structuring process of deep matter is achieved by the contribution of what he called "informatter", which is actually information at the fundamental level, and which can be described from the phenomenological point of view as a tendency towards a certain direction and purpose, and which determines a sense and an orientation to matter (Draganescu, 1990, 2004). According to this conception, the material structures are formed as follows:

$$
\text { Deep matter }+ \text { Information }=>\text { Material Structures (Matter) }
$$

These concepts have been the first to signal and define information as a fundamental element in the structuring process of matter and of the universe. Today, we dispose of many evidences that reinforce these concepts, showing that information is one of the fundamental and essential components of matter. For instance, the physicist Tegmark (2014) argued that the universe can be described actually under its last form as a mathematical universe, a universe of laws and relationships, that is, as an informational system (Tegmark, 2014). James Gate also claims that rules similar to that of the browser error codes can be obtained if some fundamental mathematical equations are rewritten under informational form, by using $(0,1)$ binary informational system (Gates, 2010), where the decision "Yes/No" unit operates (Gaiseanu, 2016a; 2016b). The obtained results are extended to living structures, suggesting that nature disposes of necessary informational ingredients to maintain the genetic cellular codes and thus to fix the possible errors. But, perhaps the most spectacular recent result is given by the experiments with microparticles in quantum mechanics, showing that 
the information of a particle, or a group of particles, can be separated from matter (Denkmayr et al., 2013; Aharonov et al., 2013). This remarkable discovery supports the reference to information as to a distinct, fundamental component of structured matter. Moreover, the theory that the particle mass of the bodies is due to the interaction with a particular (Higgs's) field (Anthony, 2012), certifies actually that mass is not an implicit quality of matter, indicating that we need to deeply revise our conception about the universe in which we live in.

Let us look now at the relationships described above. Relation (1) shows that there are two fundamental components, namely matter, as a profound, deep layer and information as a structuring agent. To analyze Relation (1) by a suggestive example, we will consider a solid body, specifically of silicon, which is the basic semiconductor material for the manufacture of microelectronic circuits and micro-systems. Although it appears to be solid, silicon, like other crystalline solids is composed by atoms, minuscule particles of the same type, which are organized under a well-defined geometric lattice. The energetic bonds between the atoms are so powerful that this body seems to have a compact, robust structure. The information contained in this body is implicit, assuring the structure itself, although this is actually built by small atoms. If an atom breaks out its position, becoming free and generating a vacancy (Gaiseanu, 2013), the configuration of the system changes and then it generates information. If the vacancy recombines with a free atom in the lattice, occupying another position (Gaiseanu, 2017a), new information is generated. If an electron descends to an inferior energy level, then a photon, so information is emitted, because the system configuration was changed (Draganescu, 1972). We can therefore observe that information is contained intrinsically in this unitary system, and is revealed when the system is reconfigured, changing its entropy. These processes include a change of matter, energy and also of information. Therefore, as stated by Relation (1), information can structure matter, but according to the previous comments, a structured system can break into information and matter, so Relation (1) may express also a reverse, destructuration process.

\section{Information Assisting the Living Structuration and Adaptation}

According to Draganescu's (1990) philosophical model, life is supported by a more complex structure, requiring more information than non-living materials, and therefore the living structures can be formally described by the relationship:

$$
\text { Material Structures (Matter) + Information => Living Structures }
$$

Living implies a complex dynamic system, incorporating much more information, as expressed in Relation (2). Living structures incorporate a specific development informational program, managing their growth to maturation, procreation and then to the involution, as a final stage of life cycle (Gaiseanu, 2019a). The living structures are able to modify their shape during their life cycle, to multiply their composing cells, and the superior categories of living structures can move themselves, that implying a high quantity of information which these systems can operate and manage. The living systems change with the environment not only information, but also matter and energy, being reactive systems, i.e., they are capable of reacting and adapting themselves according to the environmental conditions with a main scope: their survival. This means that the living systems possess the ability to detect information, distribute it within the structure, reconfigure its own structure according to needs for adaptation, and to emit information. 
The physicist Mikio Kaku (2014) classified the living creatures in four categories: (1) plants, which are immobile, reacting under minimal conditions of temperature, light, and humidity; (2) insects, with a reduced reactive system, but capable of moving; (3) mammals, with a strong reactivity, able of moving and living in organized communities; and (4) human, the most advanced living form, able not only to organize his life inside the communities, but also to design the future, according to his necessities. Relationship (2) is therefore dependent on the complexity and development level. The intrinsic information did not only refer to the interconnections between atoms, molecules, or particle collectivities of the same type, but to the connections between a large number of different species, not necessarily solid, but under various forms of aggregation. And what is maybe the most important, the living structures disposes of their own system of capture, processing and communication with their own body and with the external environment.

According to the above discussion, the body structuration is therefore assured by a more complex information system, capable of capture/processing and transmission of information, permanently correlated with itself and with the dynamics of the external environmental conditions. Reflecting on the specific organization and operating mode of living systems, we can realize that the life assurance presupposes a mechanism by which the living structures oppose to the entropy law that condemn matter to destruction, as predicted by the law of the increasing entropy within a matter system, so they should dispose of an anti-entropic, anti-destruction mechanism. In other words, the living structures seem to be managed by an internal self-organizing and self-regulating "software" that implicitly supports the material system of life, the body, and which prevents its depreciation.

This mode of operation, according to recently reported results (Gaiseanu, 2016a), is supported by an anti-entropic force, emanating from dark matter, assimilated to antimatter (Hajdukovic, 2010; 2011; 2012). According to this model, the universe itself is actually a binary information system, of the form of "Yes/No" configuration, in which matter and antimatter are two components with anti-symmetric properties (Gaiseanu, 2016a). Therefore, while matter is associated with the gravitational force, recently considered by physicists as an entropic one (Verlinde, 2010), antimatter acts on matter with an anti-gravitational force (Hajdukovic, 2010; 2011; 2012) and therefore exerts on matter an anti-entropic force (Gaiseanu, 2016a). This means that the assurance of a local equilibrium is achieved by the competition between two forces of an opposite type, like "Yes/No", action/reaction, gravity/anti-gravity, entropy/anti-entropy, and attraction/rejection. It follows therefore that the universe itself appears to be a binary informational system, which should exerts a direct effect on the development and operating mechanisms of the living structures.

\section{To an Information Science of Consciousness}

Although the concept of information has penetrated with enough difficulty in the sciences (Meijer, 2013a) and especially in the investigation and modeling field of consciousness (Gaiseanu, 2018d), today it becomes more and more well-defined and understood concept as the science advances. The Informational Model of Consciousness (IMC) discussed above is an example to see how the physics formulation on the basis of the modern physics concepts and those of science and technology of information, find their successful application. The extra-power properties of the mind, treated as belonging to an almost totally intangible world by the exact sciences, begin to find suitable explanation, to be debunked and brought to a common understanding level (Gaiseanu, 2016a; 2016b; 2017b; 2017c). 
IMC successfully can explain the mind properties related to the environmental connection and to the body connectivity. Indeed, the NDEs and associate phenomena (Gaiseanu, 2017b), PSY, ESP experiences (Gaiseanu, 2017b; 2019d) and RMEs (Gaiseanu, 2019c; 2019d) could be understood by means of informational concepts introduced by IMC in an unitary and coherent way, in close relation with the universe and its physical properties, as it was also recently explained (Gaiseanu, 2019d). Besides these relations, the entanglement phenomena, as a basic mechanism believed to support the remote communication (Radin, 2006) is actually also an informational process, as it pointed out above. In mathematical form, the informational processes can be expressed by means of probabilistic relations, using the concepts of entropy and information as initially proposed (Shannon, 1948), and/or variants, as in recent applications describing the integration of information within the various informational levels of the brain (Tononi, 2004).

Within IMC, the connectivity of mind with the body was explicated recently in terms of information by means of mechanisms of embodiment/disembodiment of information (Gaiseanu, 2019c). The intra and inter-communication of cells of the human body can be also described in terms of information (Gaiseanu, 2019c). The internal interconnectivity of cells can be understood today by means of the analogy with the digital accelerated networks in our present communication microelectronic systems, to assure the internal informational processes in the human organism as a whole (Gaiseanu, 2019c). The internal communication of a single cell, necessary from the physiological point of view to maintain its identity, can be also described in terms of information, as defined by science of information, taking into account the specific informational concepts and definitions (Gaiseanu, 2019c). The epigenetic processes, based on the mechanisms of embodiment/disembodiment of information, revealing the interaction between information (specific for the mind operation) and the material body, can explain both the short-term and large-term memory, as well as the transmission of the traits acquired during the life to the next generation, without affecting the genetics of the species (Gaiseanu, 2019c). These results show that the specific tools of information science, concerning the modeling and mathematical analysis, related by entropy, quantity of information, info-transmission parameters and other specific ones could be successfully applied in this field.

The inter-human connectivity is also an informational process, requiring a source, a transmission channel, and a receiver, like in the microelectronic informational systems. The cognitive sentient exploration of reality (CSER), demonstrated recently to be an efficient tool of knowledge, allowed to ancient philosophers to understand the universe and humans and to elaborate suitable models. The cognitive "hard" sciences and arts, as well as the educational, health, strategic and intelligence services, managerial, control, social, and even private activities are areas of applicability of CSER informational tool (Gaiseanu, 2019b).

\section{The Correspondence With Some Ancient Philosophic Models}

On the long way of searches, the philosophy rejoins today with the modern sciences. The opposite "Yes/No" units are actually the basic elements of informational systems, measurable in Bits, and consciousness is in fact the product of the informational system of the organism, described by the seven informational components, as shown above, connected by IC pole to informational universe. Indeed, the proposed bipolar model of the universe as an informational system (Gaiseanu, 2016a; 2016b; 2017b; 2017c) finds its support not only in the recent discoveries of astrophysics concerning dark matter, but also in day by day decisional activities (Gaiseanu, 2018c; 2019a). 
By its characteristics hardly to be observed and undetectable directly, dark matter has been converted in an intensive study objective. Today, it is largely accepted that dark matter does exist in large quantities in our universe (within a ratio more than 5:1 with respect to observable matter), with demonstrated effects, besides others, on the structuration process of galaxies (Freese, 2017). Indeed, the stability of galaxies could not be maintained if a high quantity of dark matter would not compensate the centrifugal effect of their rotation. Therefore, with effects also in the structuring of the life entities and on consciousness properties, as has been recently argued (Gaiseanu, 2016a; 2017b; 2017c). Thus, the old Taoist Chinese philosophy on the Yin/Yang energetic unit of contrary components, applied with great success in the unconventional meridians-based therapies in Chinese medicine (Odoul, 2014), finds its confirmation. On the other hand, this philosophy, applied for millennia even without a rigorous scientific explanation, but showing obvious positive results, confirms actually the proposed IMC, according to which matter is structured and maintained as an "informed matter" by the contribution of an anti-entropic force.

By means of IC gate concept, it could be found also a natural explanation of the religious philosophies, supported by a human high sensitivity to investigate his environment by his own CSER tool (Gaiseanu, 2019b). Indeed, these religious philosophies place the living favorable forces in the "heavens" (according to this present model being emanated by the anti-entropic force, protecting the living) and the contrary force, emphasized as the "hell", in the depths of the earth (which would correspond with the entropic, destructive force of gravity). The "immortality" problem of the soul (Meijer, 2013b; Hameroff \& Chopra, 2017), could be also approached by IMC, starting and based on the consciousness connection to IC, specifically to the anti-entropic field, which can maintain the separated informational entity after the final disembodiment of accumulated information during the life, from the physical body (Gaiseanu, 2018a). Therefore, the religious and mystical experiences (RMEs) could be explained also by concepts of defined and developed within IMC.

Philosophical concepts that have been enounced since antiquity by masters of philosophy, like Aristotle and Plato remain valid till today, constituting a starting point for the analysis and even the simulation of complex mechanisms, such as the acquisition of information in the brain (Perlovski, 2001). And not only that: masters thinkers and philosophers who have intuited and understood the functioning fundaments of consciousness, far exceeding the stage of their epoch, by using suggestive examples in the absence of the modern concepts on matter and life known today, succeeded not only to capture the attention of their own spiritual disciples and followings, but also that of some large communities of people, transforming their vision into religion. Love, equilibrium, peace, and conciliation, as IMC promotes observing the natural associative role of love force assisting the living structuration, have become more and more some generally-accepted values of life, replacing the hatred and confrontation. The qualities that we proudly call today as human/humanity and humanitarian values (Gaiseanu, 2019d) have gained more and more a duly and welcome application within the consolidated democracy societies.

The prominence of the seven centers of consciousness within the described IMC is strikingly similar to the seven chakras in some oriental philosophies. The chakras location on the structure of the human body coincides with the regions of maximum nervous activity associated with each center defined within our IMC. As in the previous cases, although unobserved directly by the sciences because of the lack of technical tools capable of reveal them, the chakras' existence is accepted and experienced with incredible results not only in the oriental, but also in the occidental world, exposing more and more and in deeper details the latent and powerful forces of our organism (Ignatenko, 1994). 
The IMC discussed in this work shows a consistency and correspondence with the most known and practiced philosophic concepts and models, representing a possible launching platform for new research directions, concerning not only the human consciousness field, but also that of the living structures in general, as we know today as a large variety of forms. IMC can open also new opportunities for the application as an individualized therapy method within the medicine procedures for the recovery of various mind disorders (Gaiseanu \& Graur, 2018), opening also new perspectives for the brain simulation.

\section{Conclusions}

Starting from the philosophical concepts on information, information-structured matter, and information assisted living structures, we can see today that these concepts have been validated by the modern sciences, based especially on the recent discoveries in quantum physics and astrophysics. Information is considered today a physical parameter, with a well-defined and distinctive statute, not only in microelectronic systems as informational tools, but also as a hidden fabric of the matter and living structures. On this basis, and demonstrating in the same time the power of the informational concepts proposed earlier by philosophy, particularly that associated with the analysis of matter and living structures proposed by Draganescu, the informational model of consciousness discussed in this work succeeds to describe not only the natural properties of consciousness, as reflected in everyday life, through the cognitive centers Ib, Ik, Iw, Il, Ia, Ic, and Icd, but also the extra-properties of mind, experimentally revealed by NDEs, ESP, PSY, and RMEs. The defining and introducing of appropriate information concepts have been allowed a coherent and unitary description of consciousness as an integrated informational system, consisting in seven components, connected to sensors and executing elements as informed matter.

The human being appears thus to be a bipolar informational/matter structure, processing the information received from the outside of the body by the informational input and reacting by output responses. As an informational-type processor, the informational system of the organism, reflected in consciousness by seven cognitive centers, is characterized by two info-inputs and two info-outputs. The info-dynamics described by IMC shows that the "free"-type information (non-related with body matter) is processed as a virtual information within the operative informational system to offer an immediate info-output reaction by attitude, while the matter-related information is processed by the automatic informational system, to transmit it as a genetic info-output. The genetic information received from the parents is represented by the genetic info-input, assuring the body development according to the age. The analysis of the info-dynamics within the seven informational subsystems shows that the degree of info-integration increases from the free-related informational subsystems to the maximum encoded information as genetic information. In terms of cognitive centers, the degree of info-integration increases from Ib and Ik to Ic and Icd. The epigenetic mechanisms explaining both the traits transmission to the next generation and short- and long- term memory by mechanisms of embodiment/disembodiment of information, reveal the intimate processes relating the information (specifically operated by the mind) and the material body, so in this way the philosophic "mind-body" and "hard" problem could find valid answers behalf of the science of information, to their debated questions

IMC can fully describe both external and internal info-connectivity in terms of information science, definitely demonstrating that the information science' tools, both regarding the analysis and modeling capabilities and the operation with the available mathematical apparatus, expressing the quantity of information and associated entropy functions, can be successfully applied in this field. The CESER defined within IMC is 
also an appropriate tool of investigation and evaluation in sciences and arts, allowing suitable applications in the managing, industrial, health, educational and intelligence, and strategic activities.

Regarded from the perspective of such a model, which is based on recent scientific results of physics, the philosophical concepts and philosophical thinking systems on the universe and life, such as the Chinese Yin/Yang philosophy, the oriental concepts on chakras-based consciousness, seem to be coincident with IMC in the essential aspects. Therefore, the high covering ranging of this informational model, deduced on the basis of the most recent scientific discoveries, and supported by these millenarian experienced philosophical models, in the same time confirming them, grants to it a major relevance in understanding of consciousness and opens new and confidence gates for future researches on this subject.

The informational model of consciousness presented here shows also that the Science and Technology of Information, this multidisciplinary and modern science, could substantially contribute not only to the spectacular development of communications, intelligent systems, robotics and more recently of non-invasive investigation tools of the brain, but also with the necessary concepts and methodologies to analyze and modeling of living systems, allowing a better and deeper understanding of consciousness.

\section{Acknowledgments}

The author dedicates this work to the Science and Technology of Information, to the founder of the Science and Technology of Information Mihai Draganescu, to Academy of Romanian Scientists and Romanian Academy of Science.

In the memory of his exemplar and eminent parents, the distinguished (Magna cum Laude) Professor Emanoil Gaiseanu and the distinguished (Emeritus) Professor Florica Gaiseanu.

\section{References}

Aharonov, Y., Popescu, S., Rohrlich, D., \& Skrzypczyk, P. (2013). Quantum Cheshire Cat. New Journal of Volume Physics, 15, 1-5. Retrieved from http_iopscience.iop.org_1367_2630_15_11_113015_art.pdf

Anthony, S. (2012). CERN: We have found the Higgs boson “God particle”. Retrieved from https://www.extremetech.com/extreme/132274-cern-we-have-found-the-god-particle

Carroll, S. (2015). From eternity to here, the quest for the ultimate theory of time. GB: One World Publication.

Chalmers, D. (1995). Facing up to the problem of consciousness. Journal of Consciousness Studies, 2(3), 200-219.

Denkmayr, T., Geppert, H., Sponar, S., Lemmel, H., Matzkin, A., Tollaksen, J., \& Hasegawa, Y. (2013). Observation of a quantum Cheshire Cat in a matter wave interferometer experiment. Retrieved from http://www.arxiv.org/pdf/1312.3775

Draganescu, M. (1972). Electronica corpului solid (in Romanian) (Electronics of the Solid State). Bucharest: Tehnica.

Draganescu, M. (1979). Profunzimilelumiimateriale (in Romanian). Bucharest: Editura Politica.

Draganescu, M. (1990). Informatiamateriei (in Romanian) (Information of matter). Bucharest: Romanian Academy.

Draganescu, M. (1997). The depth of existence (translation in English). Bucharest: Pol. Retrieved from http://www.racai.ro/books/doe

Draganescu, M. (2004). The fundamental phenomenological information of the universe. Proc. of the Romanian Academy, 5(1), 51-58.

Fracasso, C., \& Friedman, H. (2011). Near-death experiences and the possibility of disembodied consciousness: Challenges to prevailing neurobiological and psychosocial theories. Neuro Quantology, 9(1), 41-53.

Freese, K. (2017). Status of dark matter in the universities. International Journal of Modern Physics, 26(6), 1730012-1-173001231.

Gaiseanu, F. (2013). Contributions to the modelling and simulation of the atomic transport processes in silicon and polysilicon and applications. Proceedings of the Romanian Academy, 4(4), 376-384. Retrieved from http://www.acad.ro/sectii2002/proceedings/doc2013-4/15-Gaiseanu.pdf

Gaiseanu, F. (2016a). Consciousness as informational system of the human body consciousness and life physics. Cosmology and 
Astrophysics Journal, 16(1), 14-25. Retrieved from http://physics.socionic.info/index.php/physics/article/view/227/182

Gaiseanu, F. (2016b). Informational subsystems of the consciousness. Retrieved from http://www.consciousness.arizona.edu/documents/TSC2016_BOOK_of_Abstracts_for_emailWeb.pdf

Gaiseanu, F. (2017a). Modelling and simulation of the impurity diffusion and related phenomena in silicon and polysilicon systems in microfabrication and micromachining technologies. Annals of the Academy of Romanian Scientists, Series on Science and Technology of Information, 10(1), 41-78. Retrieved from http://aos.ro/wp-content/anale/IFVol10Nr1Art.4.pdf

Gaiseanu, F. (2017b). Quantum-assisted process of disembody under near-death conditions: An informational-field support model. Neuro Quantology, 15(1), 4-9. Retrieved from http://www.neuroquantology.com/index.php/journal/article/view/971

Gaiseanu, F. (2017c). An information based model of consciousness fully explaining the mind normal/paranormal properties. Neuro Quantology, 15(2), 132-140. Retrieved from https://www.neuroquantology.com/index.php/journal/article/view/1040

Gaiseanu, F. (2018a). Near-death experiences and immortality from the perspective of an informational modeling of consciousness. Gerontology \& Geriatrics Studies, 2(3). Retrieved from: https://crimsonpublishers.com/ggs/pdf/GGS.000538.pdf

Gaiseanu, F. (2018b). An informational modeling of consciousness and cognitive centers. Proc. of the Human Project Brain (HPB) International Conference: Understanding Consciousness, a scientific Quest for the 21st Century, 21-22 June, Barcelona, Spain.

Gaiseanu F. (2018c). Destiny or free will decision? A life overview from the perspective of an informational modeling of consciousness Part II: Attitude and decision criteria, free will and destiny. Gerontology \& Geriatric Studies, 4(1), 1-7. Retrieved from https://crimsonpublishers.com/ggs/pdf/GGS.000576.pdf

Gaiseanu, F. (2018d). Information: from philosophic to physics concepts for informational modeling of consciousness. Philosophy Study, 8(8), 368-382. Retrieved from http://www.davidpublisher.org/Public/uploads/Contribute/5c06323653cd2.pdf

Gaiseanu, F. (2019a). Destiny or free will decision? A life overview from the perspective of an informational modeling of consciousness Part I: Information, consciousness and life cycle. Gerontology \& Geriatric Studies, 4(1), 1-7. Retrieved from https://crimsonpublishers.com/ggs/pdf/GGS.000586.pdf

Gaiseanu, F. (2019b). Language patterns and cognitive-sentient reality: Certainty/uncertainty in cognitive-sentient exploration of reality. Hershey PA: Stephen Brock Schafer.

Gaiseanu, F. (2019c). The informational model of consciousness: Mechanisms of embodiment/disembodiment of information. Neuro Quantology, 17(4), 1-17. Retrieved from https://www.neuroquantology.com/index.php/journal/article/view/2009

Gaiseanu, F. (2019d). Human/Humanity, consciousness and universe: Informational relation. Neuro Quantology, 17(5), 20-30. doi: 10.14704/nq.2019.17.05.2122

Gaiseanu, F., \& Graur, A. (2018). Cognitive centers related attitude: Application for an iterative evaluation method in music-based therapy process. Abstract Book of the Conference on Science of Consciousness, April 2-7, Tucson, Arizona.

Gates, S. Jr. (2010). Symbols of power. Physics World, 23, 34-39.

Hajdukovic, D. (2010). Dark matter, dark energy and gravitational properties of antimatter. Retrieved from https://arxiv.org/abs/0810.3435

Hajdukovic, D. (2011). Black holes and gravitational properties of antimatter. Advanced Studies in Theoretical Physics, 5, 2-17.

Hajdukovic, D. (2012). Quantum vacuum and dark matter. Astrophys. Space Sci., 337(6), 9-14.

Hameroff, S. (1988). Quantum computation in brain microtubules? The Penrose-Hameroff “Orch OR” Model of Consciousness Philos. Trans. Royal Society London. A., 356, 1869-1898.

Hameroff, S., \& Chopra, D. (2017). Can science explain the soul? Retrieved from https://www.huffingtonpost.com/deepak-chopra/can-science-explain-the-s_b_675107.html?guccounter=1

Hameroff, S., \& Penrose, R. (1996). Orchestred reduction of quantum coherence in brain microtubules: A model for consciousness. Math. Comput. Simul, 40, 453-480.

Hameroff, S., \& Penrose, R. (2014). Consciousness in the universe: A review of the "Orch OR" Theory. Physics of the Life Review, 1, 39-79.

Haward, R. (2016). Dualism: Stanford Encyclopedia of Philosophy. Retrieved from https://plato.stanford.edu/entries/dualism/ Ignatenko, A. (1994). Cum sadevii un fenomen (in Romanian) (How to become a phenomenon). Bucharest: Biodova.

Kaku, M. (2014). The future of the mind: The scientific quest to understand enhance and empowered the mind. London: Penguin Random Hose Companies.

Meijer, D. (2013a). Information: What do you mean? On the formative element of our universe. Syntropy, 3, 1-49.

Meijer, D. (2013b). Immortality: Myth or becoming reality? On the conservation of information. Syntropy, 3, 168-207. 
Odoul, M. (2014). The complete physical pain guide-Psycho-therapeutic therapies, preface by Dr. Medynski, Thierry. Bucuresti:

Paralela. Retrieved from https://caleaechilibruluii.files.wordpress.com/2014/04/michel-odoul-ghidul-complet-al-durerilor-fizice.pdf Perlovski, L. (2001). Neural networks and intellect: Using model based concepts. Oxford: University Press.

Radin, D. (2006). Entangled minds, extrasensory experiences in a quantum theory. London: Paraview Pocket Books.

Shannon, C. (1948). The mathematical theory of communication. Bell Syst. Tech. J., 27, 379-423

Tegmark, M. (2014). Our mathematical universe: My quest for the ultimate nature of reality. USA: Randomhouse.

Tononi, G. (2004). An information integration theory of consciousness. BMC Neuroscience, 5(42).

Van Lommel, P. (2006). Near-death experience, consciousness, and the brain: A new concept about the continuity of our consciousness based on recent scientific research on near-death experience in survivors of cardiac arrest. World Futures, 62, 134-151.

Verlinde, E. (2010). On the origin of gravity and the laws of Newton. Retrieved from http://www.arXiv:1001.0785v1[hep-th] 